\title{
WallProtDB, a database resource for plant cell wall proteomics
}

Hélène San Clemente $e^{1,2}$ and Elisabeth Jamet ${ }^{1,2^{*}}$

\begin{abstract}
Background: During the last fifteen years, cell wall proteomics has become a major research field with the publication of more than 50 articles describing plant cell wall proteomes. The WallProtDB database has been designed as a tool to facilitate the inventory, the interpretation of cell wall proteomics data and the comparisons between cell wall proteomes.

Results: WallProtDB (http://www.polebio.Irsv.ups-tlse.fr/WallProtDB/) presently contains 2170 proteins and ESTs identified experimentally in 36 cell wall proteomics studies performed on 11 different plant species. Two criteria have to be met for entering WallProtDB. First one is related to the identification of proteins. Only proteins identified in plant with available genomic or ESTs data are considered to ensure unambiguous identification. Second criterion is related to the difficulty to obtain clean cell wall fractions. Indeed, since cell walls constitute an open compartment difficult to isolate, numerous proteins predicted to be intracellular and/or having functions inside the cell have been identified in cell wall extracts. Then, except proteins predicted to be plasma membrane proteins, only proteins having a predicted signal peptide and no known intracellular retention signal are included in the database. In addition, WallProtDB contains information about the strategies used to obtain cell wall protein extracts and to identify proteins by mass spectrometry and bioinformatics. Mass spectrometry data are included when available. All the proteins of WallProtDB are linked to ProtAnnDB, another database, which contains structural and functional bioinformatics annotations of proteins as well as links to other databases (Aramemnon, CAZy, Planet, Phytozome). A list of references in the cell wall proteomics field is also provided.
\end{abstract}

Conclusions: WallProtDB aims at becoming a cell wall proteome reference database. It can be updated at any time on request and provide a support for sharing cell wall proteomics data and literature references with researchers interested in plant cell wall biology.

Keywords: Cell wall, Mass spectrometry, Plant, ProtAnnDB, Proteomics

\section{Background}

The plant cell wall is an external matrix containing polysaccharides and proteins. The interest in plant cell wall proteomes has been increasing during the last years with the discovery that plant cell walls are dynamic compartments constantly modified during development and in response to environmental cues [1,2]. The physiology of plant cell walls is strongly linked to its enzyme and structural protein content. The full description of the proteins present in various cell walls at precise stages of development or in response to biotic and abiotic stresses

\footnotetext{
* Correspondence: jamet@lrsv.ups-tlse.fr

'Université de Toulouse; UPS; UMR 5546, Laboratoire de Recherche en Sciences Végétales, BP 42617, F-31326 Castanet-Tolosan, France

${ }^{2}$ CNRS; UMR 5546, BP 42617, F-31326 Castanet-Tolosan, France
}

is now a main goal for many laboratories [3-5]. Besides, the search for procedures efficiently deconstructing cell walls to produce bioethanol has renewed the interest in cell wall physiology and especially in proteins playing roles in the remodeling of cell wall polysaccharides which are the major constituents of biomass [6-9].

Recent progresses in mass spectrometry (MS) technologies have led to the identification of cell wall proteins (CWPs) allowing the description of many cell wall proteomes. The next challenge is to gain biological messages out of these data. The first problem is the validation of the proteins identified as bona fide CWPs. This point is critical in plant cell wall proteomics. Indeed, it is difficult (i) to extract proteins by non-destructive methods avoiding the leakage of plasma membranes and the release of 
intracellular proteins and (ii) to purify cells walls because they form an open compartment which is not delimited by membranes $[3,10]$. Two kinds of methods have been employed: non-destructive methods consist in the analysis of extracellular fluids collected by vacuum infiltration of different types of solutions or of culture medium; destructive methods comprise several steps starting with the grinding of plant material followed by the purification of cell walls and the extraction of proteins with salt solutions [3]. The type of identified proteins and the ratio between identified proteins predicted to be secreted and identified leaderless proteins depends on the type of method used and on the type of plant material $[10,11]$. The issue of the non-canonical CWPs, i.e. proteins having no predicted signal peptide, has been a matter of debate since the first cell wall proteomics studies [12-14]. The second problem is the quality of functional annotations of proteins in databases. They are often not sufficiently reliable to allow an appropriate biological interpretation of proteomics data because they are mostly based on sequence comparisons $[15,16]$. The third problem occurs with plants for which sequence data are not available. In this case, the proteins cannot be unambiguously identified. This is a major problem in plants since most cell wall proteins belong to multigene families [17]. All these difficulties make the comparison between different cell wall proteomes a challenging task.

In order to answer such questions, WallProtDB (http:// www.polebio.lrsv.ups-tlse.fr/WallProtDB/) was built in 2008 as a tool (i) to collect cell wall proteomics data, (ii) to facilitate their biological interpretation, and (iii) to allow comparisons between cell wall proteomes of different plant species.
A new version of WallProtDB has been recently launched with new tools allowing the comparison between cell wall proteomes from different organs of the same plants or from different plants. WallProtDB contains experimental published data which are manually curated and is restricted to plants for which sequence data, genomic or ESTs, are available. Protein accession numbers are linked to another database, ProtAnnDB (http://www.polebio.lrsv. ups-tlse.fr/ProtAnnDB/), which provides bioinformatics predictions of sub-cellular localization and functional domains of diverse plant proteins using programs available online [15].

\section{Construction and content Construction and updating}

The construction and the updating of WallProtDB are supported by the following steps performed to ensure a reliable database (Figure 1):

(1)Literature survey of plant cell wall proteomics papers. Selection of papers describing cell wall proteomes of plants with available sequenced genomes. Gathering of experimental data.

(2)Bioinformatic prediction of sub-cellular localization of proteins. This annotation is done using ProtAnnDB which is regularly enriched with new proteins. Depending on the plant of interest, protein sequences are from different databases (Table 1).

(3) Selection of proteins having predicted signal peptide, but no intracellular retention signal such as an ER canonical retention signal (IPR011679, http://www. ebi.ac.uk/interpro/entry/IPR011679; PS00014,

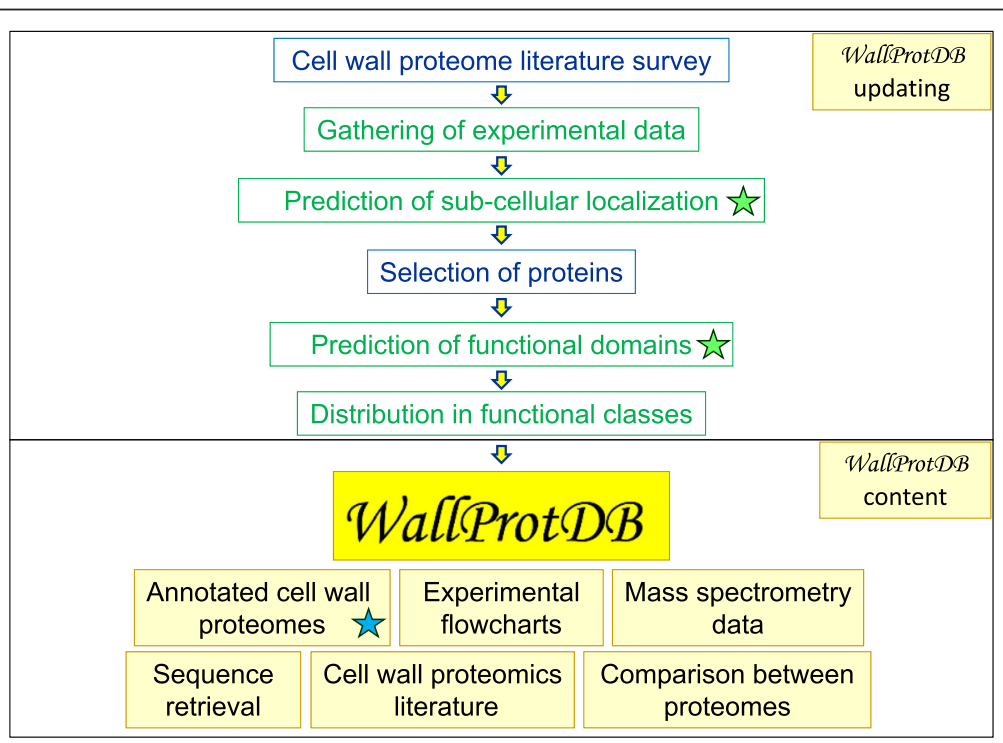

Figure 1 The WallProtDB flowchart (upper part) and content (lower part). ProtAnnDB is used at two different steps of the annotation procedure: prediction of sub-cellular localization and of functional domains (indicated by green stars). The accession number of identified protein is linked to ProtAnnDB (blue star). 
Table 1 Proteomes included in WallProtDB

\begin{tabular}{llll}
\hline Plant species & $\begin{array}{l}\text { Common } \\
\text { name }\end{array}$ & Sequence databases & Proteome size $^{\mathbf{a}^{2}}$ \\
\hline Arabidopsis thaliana & Thale cress & TAIR10 http://www.ncbi.nlm.nih.gov/ & 495 \\
Brachypodium distachyon & False brome & http://www.brachypodium.org/database & 314 \\
Brassica oleracea & cabbage & COMPBIO http://compbio.dfci.harvard.edu/index.html & 162 (ESTs) \\
Gossypium hirsutum & cotton & COTTONGEN http://www.cottongen.org/search/search_by_gene & 116 \\
Linum usitatissimum & flax & Genolin flax unigenes https://urgi.versailles.inra.fr/Species/Flax/Download-sequences & 106 \\
& & Phytozome http://www.phytozome.net/ & 199 \\
Medicago sativa & Alfalfa & JCVI: Medicago truncatula Genome Project http://medicago.jcvi.org/medicago/index.php & 199 \\
Oryza sativa & Rice & RAPD http://rapdb.dna.affrc.go.jp & 270 \\
Populus spp & Poplar & DOE Joint Genome Institute http://genome.jgi-psf.org/Poptr1_1/Poptr1_1.home.html & 142 \\
Saccharum officinarum & Sugarcane & SUCEST http://www.sucest-fun.org/index.php/overview/sucest-fun-project & 169 (ESTs) \\
Solanum lycopersicum & Tomato & sol genomics network http://solgenomics.net/ & 136 \\
Solanum tuberosum & Potato & COMPBIO http://compbio.dfci.harvard.edu/index.html & \\
\hline
\end{tabular}

${ }^{a}$ ESTs mean that protein identification was done using translated ESTs because of the lack of genomic data. Note that the distribution of proteins into functional classes is indicated in Additional file 1 for the cell wall proteome of all the plant species listed in this table.

http://prosite.expasy.org/PS00014) and no more than one trans-membrane domain as predicted by TMHMM (http://www.cbs.dtu.dk/services/ TMHMM-2.0/).

(4) Bioinformatics prediction of functional domains. This annotation is done using ProtAnnDB (see below).

(5)Definition of a dictionary for the functional annotation of proteins, based on Pfam (http://pfam. xfam.org) [18] or InterPro (http://www.ebi.ac.uk/ interpro/) [19] domain repertoires. This step ensures that the same annotation is used for all the proteins sharing the same predicted functional domains.

(6) Classification of proteins into 8 functional classes on the basis of the presence of predicted functional domains [10]: proteins acting on cell wall polysaccharides, oxido-reductases, proteases, proteins related to lipid metabolism, proteins with interaction domains (with proteins or polysaccharides), proteins possibly involved in signaling, structural proteins, proteins with yet unknown function. All the other proteins are included in a ninth class named "miscellaneous proteins" (Additional file 1).

(7)Design of a flowchart form allowing the description of most of the possible strategies usable to isolate CWPs and to identify them by MS and bioinformatics [3]. Customize the form for each set of experimental data (for an example, see Analysis of the cell wall proteome of Brachypodium distachyon young leaves: http://www.polebio.lrsv.ups-tlse.fr/ WallProtDB_data/biblio/biblio26.html).
(8) Addition of MS data when available using either the X! Tandem software [20] or links to excel sheets found as supplementary data in articles of interest. When the data are in the X!Tandem format, it is possible to visualize the sequenced peptides on the protein sequence and their MS/MS fragmentation data.

(9) Search for homologous proteins in closely related genomes when only ESTs are available. The identification of homologous genes allows completing the bioinformatics prediction of signal peptide and/or of functional domains when EST sequences are not full-length. This is the case for Saccharum officinarum and Brassica oleracea for which homologous genes have been searched for in Sorghum bicolor and Arabidopsis thaliana respectively.

(10) Addition of cell wall proteomics literature. Direct links to articles or to their abstract in PubMedNCBI (http://www.ncbi.nlm.nih.gov/pubmed) are available.

Data are stored in a mySQL database. WallProtDB is queried through a web interface constructed in the PHP code (http://www.php.net/).

\section{Bioinformatics annotation of proteins using ProtAnnDB}

ProtAnnDB is an annotation tool used for (i) selecting proteins to be included in WallProtDB and (ii) providing annotation of selected proteins. ProtAnnDB collects the results of bioinformatics predictions of sub-cellular localization and functional domains using available programs [17]. The following programs or databases have been used for prediction of sub-cellular localization: SignalP (http://www.cbs. 
dtu.dk/services/SignalP/) [21], TargetP (http://www.cbs.dtu. $\mathrm{dk} /$ services/TargetP/) [22], Predotar (http://urgi.versailles. inra.fr/predotar/predotar.html) [23], Aramemnon (http:// aramemnon.botanik.uni-koeln.de/) [24], TMHMM (http:// www.cbs.dtu.dk/services/TMHMM-2.0/), GPIsom (http:// gpi.unibe.ch/) [25] and PredGPI (http://gpcr.biocomp. unibo.it/predgpi/pred.htm) [26]. The databases used for the prediction of functional domains are Pfam [18], InterPro [19] and PROSITE (http://prosite.expasy.org/) [27]. Pro$t A n n D B$ also offers links to other databases providing genomic or gene regulation data such as Phytozome which collects genomic data (http://www.phytozome.net) and PlaNet which provides co-expression networks (http:// aranet.mpimp-golm.mpg.de/) [28]. PlaNet has been chosen because it gives information on all the $A$. thaliana genes as well as on other plant species. ProtAnnDB has also links to Aramemnon which presently contains membrane protein data for nine plant species (http://aramemnon.botanik.unikoeln.de/index.ep) and to two databases which collect expert annotation on cell wall protein families: (i) the PeroxiBase which is dedicated to peroxidases (http://peroxibase. toulouse.inra.fr/) [29] and (ii) CAZy which provides annotation of carbohydrate active enzymes (http://www.cazy.org/, http://csbl.bmb.uga.edu/dbCAN//) [30,31].

\section{Tools for browsing WallProtDB}

They are three ways to query WallProtDB: "Detailed search", "Summarized search" or "Blast search".

(1) The "Detailed search" interface offers several criteria: (1) protein accession number; (2) plant species; (3) plant material; (4) protein functional class; (5) protein family; (6) keyword. These criteria can be combined to refine comparisons. The result of the query is a customizable table that can be exported in different formats such as a tab delimited text, an excel sheet or a pdf file (Figure 2). Alternatively, they can be directly printed. Hyperlinks lead to ProtAnnDB bioinformatics annotation, experimental flowcharts and MS data (Figure 2). Protein sequences can be retrieved in FASTA format.

(2) The "Summarized search" interface provides tools for overall proteome comparisons. The result of the query is a table in which the numbers of proteins in each (i) protein functional class, (ii) protein family or (iii) protein (putative) function are indicated (Figure 3). As mentioned above, different formats are available for export of query results. It is also possible to draw a Venn diagram to visualize proteome comparisons within a plant species (Figure 4). All the figures are clickable, thus enabling retrieval of lists of the corresponding proteins.

(3) The "Blast search" [32] permits finding sequences homologous to a given nucleic or protein sequence in WallProtDB. A list of hits is proposed together with the possibility to visualize sequence comparisons and to collect the protein sequences in the FASTA format. It allows clustering newly identified CWPs with proteins present in the database. Then, it is easier to link the presence of some protein clusters to different physiological conditions and/or to cell wall types.

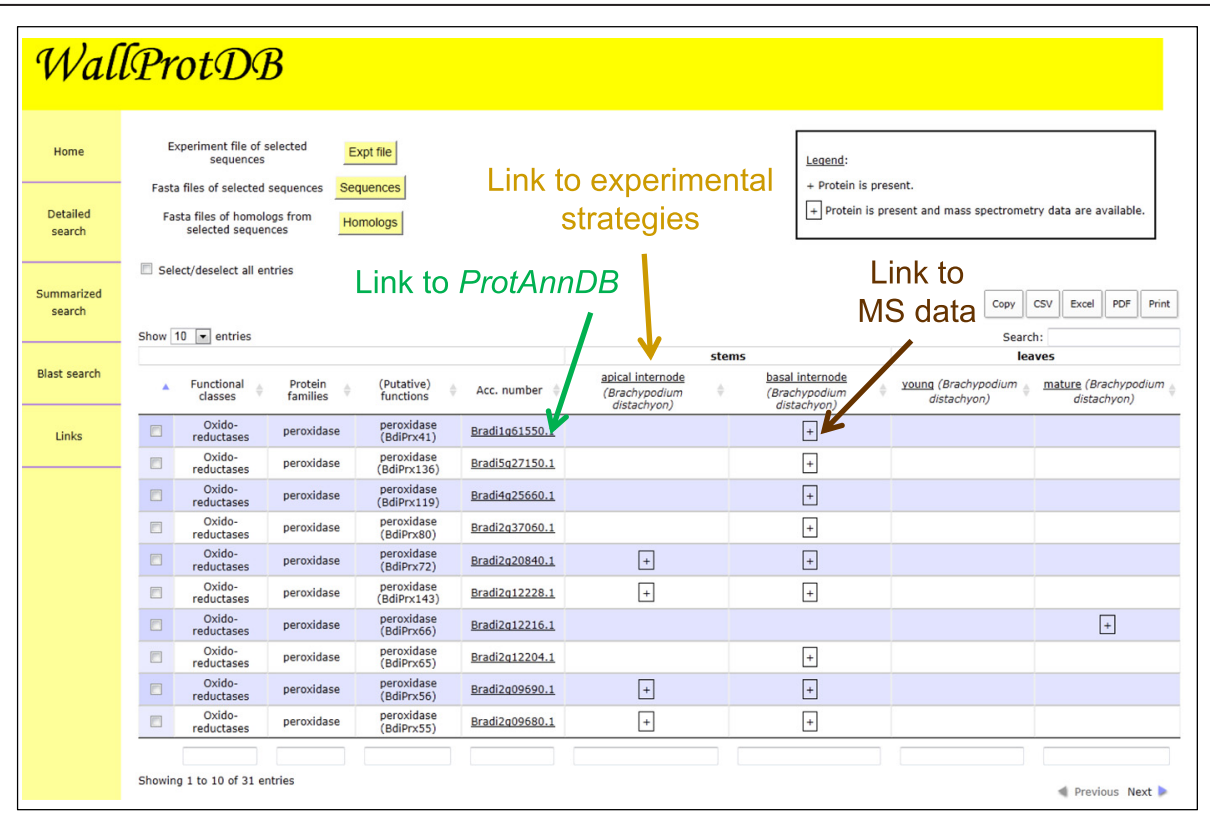

Figure 2 An example of results of a Detailed search: B. distachyon as a plant; peroxidases as a protein family; leaves and stems as plant organs. Note that only a part of the result sheet is shown. 


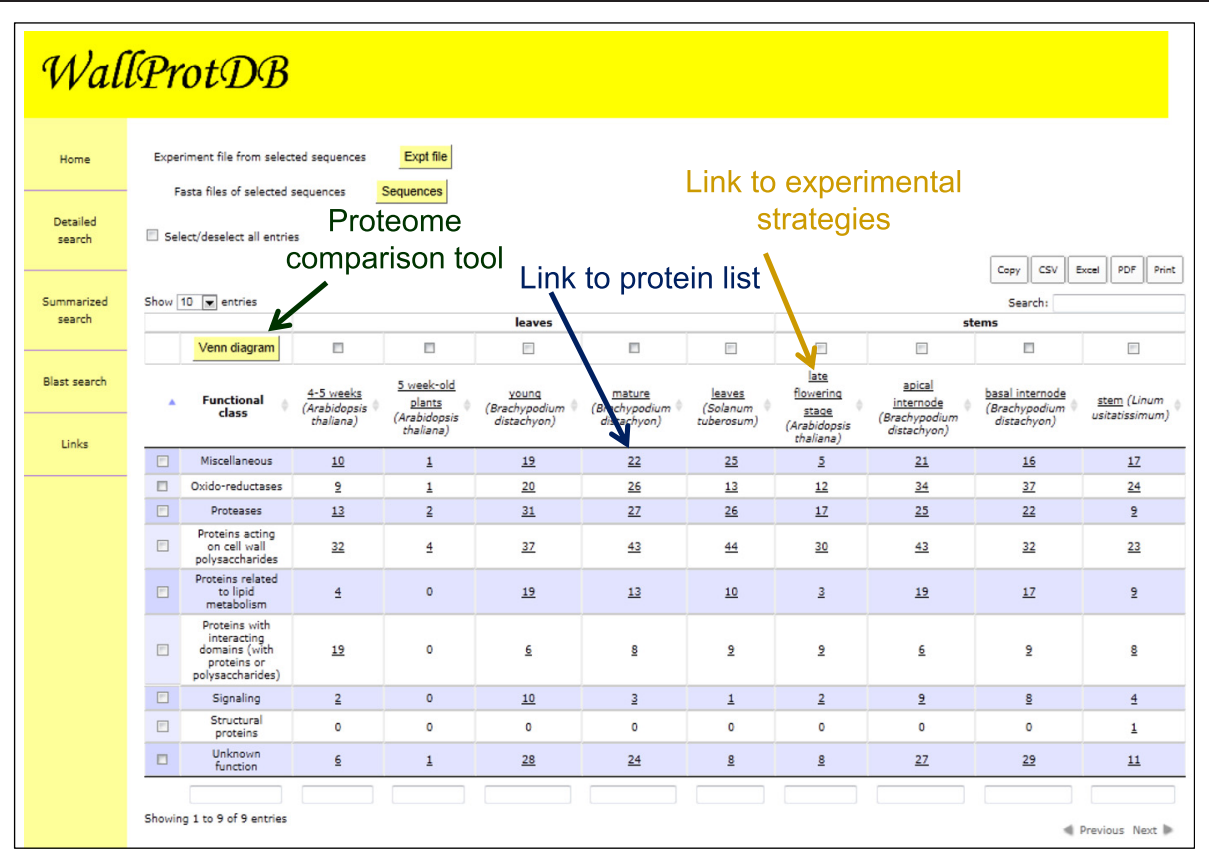

Figure 3 An example of results of a Summarized search performed using the following criteria: group by functional class; $A$. thaliana, B. distachyon, L. usitatissimum and S. tuberosum as plants; leaves and stems as plant organs.

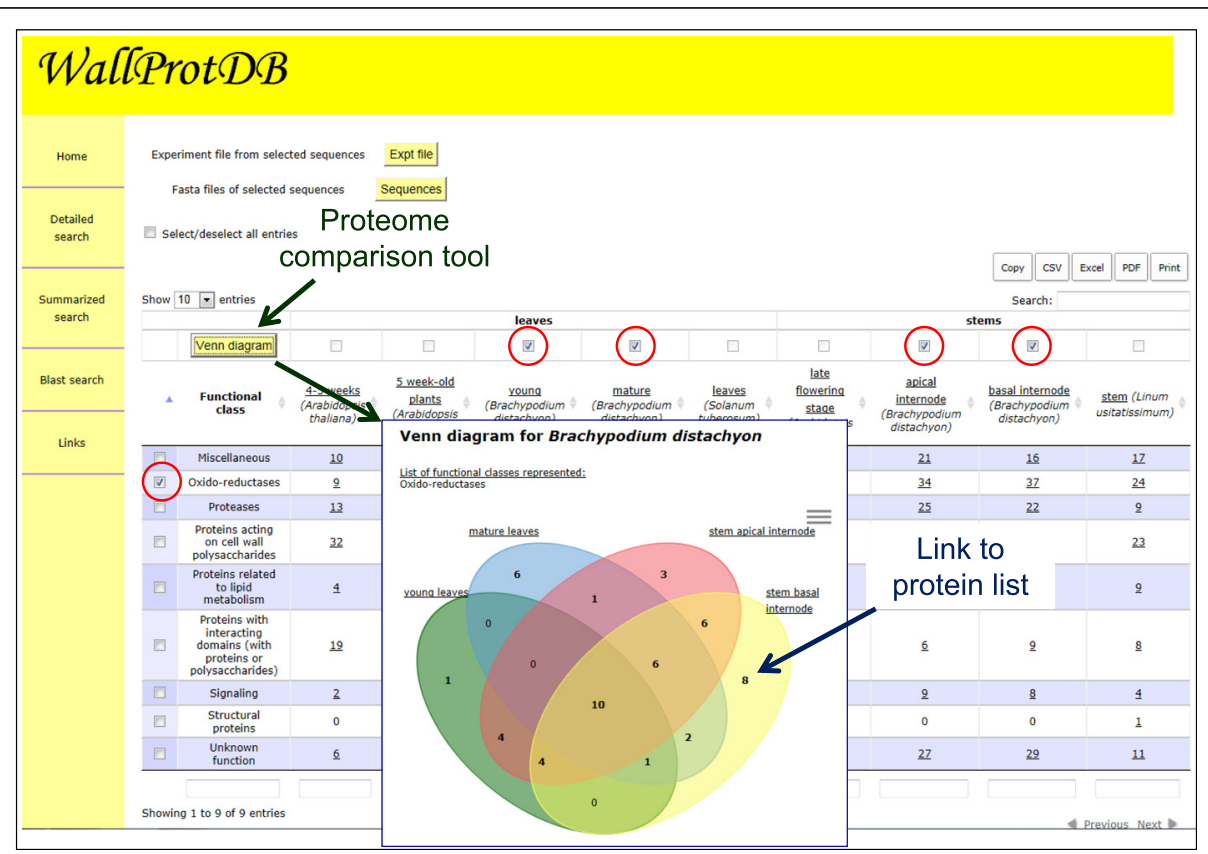

Figure 4 An example of comparison between different proteomes within a plant species using the Summarized search. The same criteria as in Figure 3 were used to obtain a table of results. Then, the Venn diagram was obtained after selection of B. distachyon as a plant, oxido-reductases as a functional class, and the four following experiments: young leaves (green), mature leaves (blue), apical internodes (pink), basal internodes (yellow). 


\section{Utility and discussion}

At present, WallProtDB contains 2170 proteins and expressed sequence tags (ESTs) identified in 36 cell wall proteomics studies performed on 11 different plant species (8 dicots and 3 monocots) (Table 1, Additional file 1). It also offers tools for comparisons between proteomes. Wall$\operatorname{Prot} D B$ is regularly updated with newly published experimental data which are manually curated to obtain a homogeneous annotation (prediction of sub-cellular localization and functional domains of proteins). Only proteins having a signal peptide to address proteins to the secretion pathway and no known intracellular retention signal are included in the database. Proteins predicted to be plasma membrane proteins have been introduced in the database such as cellulose synthase, callose synthase or receptor kinases. They have been identified through peptides located in their extracellular domain. They are not true CWPs, but since they are involved in cell wall biogenesis or in signal transduction, they might be of interest for people working in the plant biology field. In addition, WallProtDB contains information about the protocols used to obtain cell wall protein extracts and about the strategies to identify proteins by MS and bioinformatics, as well as MS data when available. Furthermore, WallProtDB provides a list of references in the cell wall proteomics field which is regularly updated and comprises experimental articles as well as reviews.

WallProtDB complements other databases such as SUBcellular location database for Arabidopsis proteins (SUBA3, http://suba.plantenergy.uwa.edu.au/) [33], The Plant Proteome Database (PPDB, http://ppdb.tc.cornell. $\mathrm{edu} /$ ) [34] and the cellwallgenomics database (http:// cellwall.genomics.purdue.edu/intro/index.html). In each of these databases, the gathering of information is done in a different way. In SUBA3, only $A$. thaliana proteins are listed and all the proteins identified in published proteomes are included. This can be misleading because proteins known to be intracellular can be found in proteomes called "cell wall proteomes". For example, the At5g38410 A. thaliana small subunit of RUBISCO is mentioned as an extracellular or a plasma membrane protein although it is a well described chloroplastic protein. However, since all the proteins identified in cell wall proteomes are listed, SUBA3 is useful to get access to leaderless proteins identified in cell wall proteomes. PPDB is devoted to A. thaliana, Oryza sativa and Zea mays. It contains experimental MS data on proteins identified in different organs or sub-cellular compartments including the cell wall. Finally, the cellwallgenomics database provides repertoires of genes involved in cell wall biogenesis in A. thaliana, O. sativa, S. bicolor and $Z$. mays including intracellular proteins such as glycosyl transferases involved in the biosynthesis of cell wall polysaccharides. It also gives information on some mutants and on techniques useful to study cell wall biology, but no cell wall proteomics data.

\section{Conclusions}

To date, WallProtDB describes the content of cell wall proteomes and proposes tools for their analysis. It contains proteins with a high probability of being bona fide CWPs with regard to our present knowledge of the secretion pathway and of cell wall physiology. However, in the future, it could also include proteins with no predicted signal peptides, but experimentally proven to be located in cell walls by alternative methods such as localization of proteins tagged with fluorescent proteins or immunolabeling [3]. So far, there are only a few examples of such proteins in plants. The symplastic mannitol deshydrogenase has been shown to be secreted upon pathogen infection, and the secretion can occur in the presence of brefeldin A. However, the mechanism of secretion has not been described [35]. The exocyst-positive organelle (EXPO) could mediate the exocytosis from cytosol to cell wall of learderless proteins such as SAMS2 (S-adenosylmethionine synthetase 2) [36]. Since all the proteins included in WallProtDB are annotated in the same way, it allows fine comparisons between cell wall proteomes of different species and of various plant materials. In addition, it allows clustering proteins on a sequence homology basis. New proteomes can be introduced in WallProtDB on request, providing EST or genomic sequences of the plant of interest are available. The distribution of proteins into functional classes will certainly evolve when the functions of the proteins are experimentally determined. New functional classes can be easily created. Finally, the possibility to introduce wall proteomes of plant pathogens or symbionts will be considered since they share common protein families with plant cell wall proteomes. Altogether, WallProtDB aims at becoming a cell wall proteome reference database.

\section{Availability and requirements}

WallProtDB is freely available at the following address: http://www.polebio.lrsv.ups-tlse.fr/WallProtDB/. It is compatible with all major web browsers.

\section{Additional file}

Additional file 1: The WallProtDB content.

Competing interests

The authors declare that they have no competing interests.

\section{Authors' contributions}

EJ has supervised the project and written the manuscript. HSC has created the WallProtDB database and is updating it regularly. Both authors have read and approved the manuscript. 


\section{Acknowledgements}

The authors are thankful to Université Paul Sabatier (Toulouse, France) and Centre National de la Recherche Scientifique (CNRS) for funding their research. They are thankful to Drs Cécile Albenne, Hervé Canut, Christophe Dunand, Valérie Pacquit, and late Pr Rafael Pont-Lezica for stimulating discussions. They also wish to thank Pr Klaas J van Wijk at Cornell University for linking WallProtDB to the Plant Protein Database (PPDB). The GenoToul Bioinformatics platform is acknowledged for providing the JQuery jvenn plug-in and facilities (http://www.genotoul.fr/index.php?id=12).

Received: 10 December 2014 Accepted: 6 January 2015

Published online: 16 January 2015

\section{References}

1. Fry SC. Primary cell wall metabolism: tracking the careers of wall polymers in living plant cells. New Phytol. 2004;161:641-75.

2. Passardi F, Penel C, Dunand C. Performing the paradoxical: how plant peroxidases modify the cell wall. Trends Plant Sci. 2004;9:534-40.

3. Albenne $\mathrm{C}$, Canut $\mathrm{H}$, Jamet E. Plant cell wall proteomics : the leadership of Arabidopsis thaliana. Front Plant Sci. 2013:4:111.

4. Lee SJ, Saravanan RS, Damasceno CM, Yamane H, Kim BD, Rose JK. Digging deeper into the plant cell wall proteome. Plant Physiol Biochem. 2004;42(12):979-88.

5. Jung Y-H, Agrawal G, Rakwal R, Jwa N-S. Secretome: toward deciphering the secretory pathways and beyond. In: Agrawal G, Rakwal R, editors. Plant proteomics: technologies, strategies and applications. Hoboken, NJ: John Wiley \& Sons Inc; 2008. p. 764.

6. Gomez L, Steele-King C, McQueenMason S. Sustainable liquid biofuels from biomass: the writing of the walls. New Phytol. 2008;178:473-85.

7. Jordan D, Bowman M, Braker J, Dien B, Hector R, Lee C, et al. Plant cell walls to ethanol. Biochem J. 2012:442:241-52.

8. Ragauskas A, Williams C, Davison B, Britovsek G, Cairney J, Eckert C, et al. The path forward for biofuels and biomaterials. Science. 2006;311:484-9.

9. Carpita N. Progress in the biological synthesis of the plant cell wall: new ideas for improving biomass for bioenergy. Curr Opin Biotechnol. 2012;23:330-7

10. Jamet E, Albenne C, Boudart G, Irshad M, Canut H, Pont-Lezica R. Recent advances in plant cell wall proteomics. Proteomics. 2008;8:893-908.

11. Albenne C, Canut H, Hoffmann L, Jamet E. Plant cell wall proteins: a large body of data, but what about runaways? Proteomes. 2014;2:224-42.

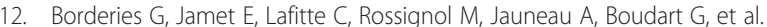
Proteomics of loosely bound cell wall proteins of Arabidopsis thaliana cell suspension cultures: a critical analysis. Electrophoresis. 2003;24:3421-32.

13. Chivasa S, Ndimba BK, Simon WJ, Robertson D, Yu X-L, Knox JP, et al. Proteomic analysis of the Arabidopsis thaliana cell wall. Electrophoresis. 2002;23:1754-65

14. Rose JKC, Lee S-J. Straying off the highway: trafficking of secreted plant proteins and complexity in the plant cell wall proteome. Plant Physiol Biochem. 2010;153:433-6.

15. San Clemente H, Pont-Lezica R, Jamet E. Bioinformatics as a tool for assessing the quality of sub-cellular proteomic strategies and inferring functions of proteins: plant cell wall proteomics as a test case. Bioinform Biol Insights. 2009;3:15-28.

16. Jamet E. Bioinformatics as a critical prerequisite to transcriptome and proteome studies. J Exp Bot. 2004;55:1977-9.

17. Yokoyama R, Nishitani K. Genomic basis for cell-wall diversity in plants. A comparative approach to gene families in rice and Arabidopsis. Plant Cell Physiol. 2004;45:1111-21.

18. Finn R, Tate J, Mistry J, Coggill P, Sammut J, Hotz H, et al. The PFAM protein families dabase. Nucleic Acids Res. 2008;36:D281-8.

19. Quevillon E, Silventoinen V, Pillai S, Harte N, Mulder N, Apweiler R, et al. InterProScan: protein domains identifier. Nucleic Acids Res. 2005:33(Web Server issue):W116-20

20. Fenyö D, Eriksson J, Beavis R. Mass spectrometric protein identification using the global proteome machine. Methods Mol Biol. 2010;673:189-202.

21. Bendtsen J, Nielsen H, von Heijne G, Brunak S. Improved prediction of signal peptides: SignalP 3.0. J Mol Biol. 2004;340:783-95.

22. Emanuelsson $\mathrm{O}$, Nielsen $\mathrm{H}$, Brunak $\mathrm{S}$, von Heijne G. Predicting subcellular localization of proteins based on their $\mathrm{N}$-terminal amino acid sequence. J Mol Biol. 2000;300:1005-16.
23. Small I, Peeters N, Legeai F, Lurin C. Predotar: a tool for rapidly screen proteomes for $\mathrm{N}$-terminal targeting sequences. Proteomics. 2004;4:1581-90.

24. Schwacke R, Schneider A, van der Graaff E, Fischer K, Catoni E, Desimone M, et al. ARAMEMNON, a novel database for Arabidopsis integral membrane proteins. Plant Physiol. 2003;131(1):16-26.

25. Fankhauser N, Mäser P. Identification of GPI anchor attachment signals by a Kohonen self-organizing map. Bioinformatics. 2005;21:1846-52.

26. Pierleoni A, Martelli P, Casadio R. PredGPI: a GPI-anchor predictor. BMC Bioinformatics. 2008;9:392.

27. Hulo N, Bairoch A, Bulliard V, Cerutti L, Cuche B, De Castro E, et al. The 20 years of PROSITE. Nucleic Acids Res. 2008;36:D245-9.

28. Mutwil M, Klie S, Tohge T, Giorgi F, Wilkins O, Campbell M, et al. PlaNet: combined sequence and expression comparisons across plant networks derived from seven species. Plant Cell. 2011;23:895-910

29. Fawal N, Li Q, Savelli B, Brette M, Passaia G, Fabre M, et al. PeroxiBase: a database for large-scale evolutionary analysis of peroxidases. Nucleic Acids Res. 2013:41(Database issue):441-4.

30. Lombard V, Golaconda Ramulu H, Drula E, Coutinho P, Henrissat B. The carbohydrate-active enzymes database (CAZy) in 2013. Nucleic Acids Res. 2013:42(Database issue):D490-5

31. Yin Y, Mao X, Yang J, Chen X, Mao F, Xu Y. dbCAN: a web resource for automated carbohydrate-active enzyme annotation. Nucleic Acids Res. 2012;40(Web Server issue):W445-51

32. Altschul SF, Gish W, Miller W, Myers EW, Lipman D. Basic local alignement search tool. J Mol Biol. 1990;215:403-10.

33. Tanz S, Castleden I, Hooper C, Vacher M, Small I, Millar H. SUBA3: a database for integrating experimentation and prediction to define the SUBcellular location of proteins in Arabidopsis. Nucleic Acids Res. 2013;41(Database issue):D1185-91.

34. Sun Q, Zybailov B, Majeran W, Friso G, Olinares P, van Wijk K. PPDB, the Plant Proteomics Database at Cornell. Nucleic Acids Res. 2008;37(Database issue):D969-74.

35. Cheng F, Zamski E, Guo W, Pharr D, Williamson J. Salicylic acid stimulates secretion of the normally symplastic enzyme mannitol dehydrogenase: a possible defense against mannitol-secreting fungal pathogens. Planta. 2009;230:1093-103.

36. Wang J, Ding Y, Wang J, Hillmer S, Miao Y, Lo S, et al. EXPO, an exocyst-positive organelle distinct from multivesicular endosomes and autophagosomes, mediates cytosol to cell wall exocytosis in Arabidopsis and tobacco cells. Plant Cell. 2010;22:4009-30.

\section{Submit your next manuscript to BioMed Central and take full advantage of:}

- Convenient online submission

- Thorough peer review

- No space constraints or color figure charges

- Immediate publication on acceptance

- Inclusion in PubMed, CAS, Scopus and Google Scholar

- Research which is freely available for redistribution
C Biomed Central 